\title{
Actores no estatales en la Antártica. Un análisis desde la Ciencia, el Medioambiente y el Turismo ${ }^{1}$
}

\author{
Non-State actors in Antarctica. \\ An analysis from Science, Environment and Tourism \\ Miguel Ángel Salazar Urrutia* \\ "Investigador Centro de Estudios Hemisféricos y Polares \\ $\bowtie$ miguelsalazaru@gmail.com
}

\begin{abstract}
RESUMEN
En una proposición revisionista de los Paradigmas de las Relaciones Internacionales, se propone un enfoque alternativo para explicar las interacciones entre los actores del Sistema del Tratado Antártico (STA), al cual se le denomina pararrealismo. En lo fundamental, este artículo, presenta una descripción y análisis sobre las actividades de los Actores No Estatales en la Antártica de acuerdo a tres aristas de reflexión y análisis: Ciencia, Medioambiente y Turismo. Estas áreas son expuestas por medios de casos concretos y particulares en su relación con Chile como actor del STA.
\end{abstract}

PALABRAS CLAVE: Pararrealismo, Sistema del Tratado Antártico, Ciencia, Medioambiente, Turismo.

\begin{abstract}
A revisionist proposition of the paradigms of international relations, proposes an alternative approach to explain the interactions between the actors of the Antarctic Treaty System (ATS), which is called pararealism. Fundamentally, this article presents a description

1 Este trabajo fue presentado en el XVIII Encuentro de Historiadores Antárticos Latinoamericanos el 06 de septiembre del 2018, y es el resultado de un trabajo final de investigación para la obtención del grado académico de Magister en Relaciones Internacionales en la Pontificia Universidad Católica de Valparaíso en el año 2018, y fue dirigido por Mauricio Burgos Quezada, titulado Actores no Estatales en la Antártica. Una aproximación a las relaciones transnacionales y sus implicancias en Chile como país antártico. Dicho trabajo comprende tres aristas de análisis no incluidas en el presente artículo, la pesca, los transportes y las telecomunicaciones.
\end{abstract}


and analysis of the activities of non-State actors in Antarctica according to three areas of reflection and analysis: science, environment and tourism. These areas are exposed by specific and particular cases in its relationship with Chile as actor in the ATS.

KEY WORDS: Pararealism, Antarctic Treaty System, Science, Environment, Tourism.

\section{INTRODUCCION}

La globalización ya es un hecho, y es uno de los principales elementos de cambio en el sistema internacional del siglo XXI, entendida como un proceso contemporáneo de las relaciones económicas internacionales, donde la mundialización de los mercados y la apertura comercial está caracterizada por la ampliación de actores, el transnacionalismo, la interdependencia, las tecnologías de la información y la comunicación, los flujos financieros y el avance de los medios de transporte (Allard, 2008, p. 22).

Ahora bien, la Antártica, a pesar de su extremo condicionamiento geográfico, no logra marginarse del alcance que tiene este proceso. Efectivamente, este continente, bajo la coyuntura de la globalización, ha visto la emergencia de nuevos Actores No Estatales (ANE), los cuales poseen distintas capacidades en recursos, tecnología e innovación, factores esenciales para el desarrollo de actividades en materias de interés como la ciencia, el medio ambiente, el turismo, las pesca, los transportes y telecomunicaciones. Además, poseen una efectiva capacidad de representación en instancias estatales del Sistema del Tratado Antártico (STA), ejerciendo voz e influyendo en el decision making de sus miembros.

En cuanto al STA, este ha transitado por coyunturas bipolares, unipolares y ahora mismo, bajo un contexto multipolar, o incluso "unimultipolar", cuyos actores se han diversificado desde Estados, individuos, fundaciones, centros de investigación, laboratorios, universidades, organizaciones medioambientales, y Empresas Multinacionales o transnacionales (EMN). Este proceso de proliferación de actores en la Antártica, sin duda, se ha conformado en absoluta sintonía con los progresos de la tecnológica en los transportes y las telecomunicaciones, así como de la liberalización de los mercados, fomentando la progresiva visita de personas provenientes, principalmente, de países desarrollados.

Esta premisa, hace ineludible el replanteamiento sobre la forma en que entendemos, por un lado el Sistema Internacional, es decir, como un conjunto de actores cuyas relaciones generan una relación de poder (estructura) dentro de la cual se produce una red compleja de interacciones (proceso) de acuerdo a determinadas reglas ( (Barbé, 2007, p. 151) , y por otro lado el STA, es decir, un subsistema semiabierto, sustentado esencialmente en el Tratado Antártico de 1959, constituido por un conjunto de convenciones e instrumentos adicionales adoptados en las Reuniones Consultivas del Tratado Antártico (RCTA) (Rothwell, 2009, p. 125), con actores diversificados, generando interacciones entre Estados, Organizaciones 
Intergubernamentales, empresas, organizaciones no gubernamentales y la sociedad civil. Estas interacciones, responden a distintos intereses en función del área a las que compete, pero normalmente se encuentran entrelazadas entre sí. En efecto, la ciencia, la protección y preservación al medioambiente, el turismo, la pesca, los sistemas de transporte y telecomunicaciones son áreas inherentes entre sí.

Por consiguiente, se considera que los paradigmas en relaciones internacionales son hijos de su tiempo, y estos evolucionan en sintonía a la amplitud de perspectivas frente a los cambios. Hoy por hoy, contamos con un amplio estado del arte de perspectivas y enfoques para concebir las relaciones internacionales. Sin embargo, el realismo sigue siendo un paradigma dominante en esta disciplina. ¡Y con justa razón! El realismo, es una práctica que permite mesurar racional y estratégicamente el uso de los recursos disponibles para la consecución de los intereses de un Estado, pero ante los profundos cambios en la realidad internacional, la consolidación de la globalización y la emergencia de nuevos actores, el realismo se muestra como una teoría poco apropiada ${ }^{1}$. Por lo tanto, ¿hasta qué punto podemos concebir el STA como un sistema interestatal? ¿Son los Estados los únicos actores en él? Muchos dirán, a ciencia cierta, que efectivamente existe una diversidad de actores en el STA, pero entonces ¿bajo qué paradigma habrá que comprender las dinámicas inter y trans nacionales que se generan en la Antártica?

De este modo, se plantea un nuevo enfoque denominado pararrealismo, que establece que los ANE pueden representar, total o parcialmente, el interés de los Estados por medios de sus actividades, movilizando recursos y ejerciendo influencia en terceros. Así, se establece un medio indirecto de poder en un territorio sin soberanía efectiva (bajo el principio westfaliano) y de gobernanza multiestatal. Además, históricamente desde la era de los descubrimientos geográficos a fines del siglo $\mathrm{XV}$, los nacientes Estados de Europa instaron a sus entidades privadas para llevar a cabo las tareas de conquista y de comercio en los territorios de ultramar, pudiendo encontrar, en tal sentido, la antesala histórica, de los medios indirectos de poder que ejercen las potencia en regiones que no necesariamente forman parte de su soberanía territorial. Esta lógica, de "realismo paralelo" es tomada como antecedente, para sustentar el enfoque teórico del presente trabajo.

En cuanto al marco metodológico del presente artículo, este se constituye en un trabajo heurístico y hermenéutico de material bibliográfico relativo a los actores no estatales y su relación con los Estados y otras organizaciones internacionales. Además, está complementado por un conjunto de entrevistas que dan testimonios desde distintos referentes en la materia de Chile, Estados Unidos y Francia.

2- El enfoque realista se sustenta en tres elementos que definen, en su versión clásica de E. H. Carr y Hans Morgenthau, la esencia de su núcleo teórico. Primero, que los Estados son los principales actores en la política internacional. Segundo, que los Estados son unitarios y racionales en el uso de medios para la consecución de sus objetivos e intereses. Finalmente, el principal interés de los Estados es el poder como medio y como fin. (Allan, 2001, p. 81). 
Ciencia para la diplomacia

Debido principalmente a las características geográficas de la Antártica, desde los albores de su descubrimiento, y posterior exploración y conquista, siempre ha sido un foco de interés científico. Por lo tanto, la ciencia se presenta como una constante en el desarrollo histórico del continente, de tal forma que la mayoría de actividades realizadas sobre estas regiones del planeta están dedicadas a esta materia. De este modo, se puede decir que la ciencia ha sido, y es actualmente, un elemento que permite mesurar el ejercicio del poder en el STA.

Es más, la diplomacia en la Antártica no solo depende de los elementos tradicionales que la componen; informar, representar y negociar, sino que las diplomacias exitosas del continente están determinadas principalmente por la actividad científica que puedan generar y/o promover sobre y en el continente (Villamizar Lamus, 2017, p. 131) por lo tanto, es un elemento primordial para el establecimiento de las relaciones diplomáticas. En efecto, Villamizar, en su obra "Más allá de las fronteras: los horizontes geopolíticos de la Antártida”, cita un interesante estudio a Dudeney y Walton titulado Leadership in politics and science within the Antarctic Treaty (2012), el cual presenta un acertado análisis comparativo sobre producción científica de working-papers publicados por los países miembros del Tratado Antártico, afirmando que ninguno de los signatarios posteriores al tratado parece jugar un papel importante en la gestión de la Antártica en comparación con este grupo (12 signatarios), ya que tan solo la mitad de todas las partes consultivas producen sólo el 7\% de los documentos de Política Antártica (Dudeney \& Walton, 2012, p. 1). Además, de la información producida por lo Estados en las RCTA, los organismos internacionales como el Scientific Committee on Antarctic Research (SCAR), la Convención para la Conservación de los Recursos Marinos Vivos (CCRVMA o CCAMLR por su siglas en inglés), el Council of Managers of National Antarctic Programs COMNAP, y organizaciones no gubernamentales y empresas de turismo a través de la Antarctic and Southern Ocean Coalition (ASOC), y la International Association of Antarctica Tour Operators (IAATO), sin ser Estados, generan la otra gran parte del total. Así, cabe hacerse el cuestionamiento sobre quién realiza esta producción científica. Si bien, se sabe que gran parte de esta actividad está destinada a los programas de cada Estado, existen vínculos con otros actores, que en gran medida generan este conocimiento, por medio del acceso a recursos provenientes desde distintas fuentes públicas y/o privadas, y que son susceptibles de ser benéficas al Estado. Desde la perspectiva de Hemmings, los Estados contratan a entidades descentralizadas o bien al sector privado para su programa antártico como históricamente lo ha hecho Estados Unidos para obtener servicios de apoyo - es decir, el Estado permanece en el asiento del conductor, a través de disposiciones legales nacionales y contractuales (Hemmings, 2014, p. 30), y los ANE serían sus pasajeros que elaboran la producción científica a beneficio de sus relaciones con otros Estados, dejando de lado la lógica realista y el principio del poder por el poder. Así, se complementan e interdependizan 
los intereses, y aunque los beneficios no son necesariamente mutuos, las relaciones públicoprivado, vinculadas al continente antártico, se hacen cada vez más comunes.

Un caso no menor, el cual se puede interpretar desde una visión pararrealista, es el Centro de Estudios Científicos (CECs) en Chile, que publicó el hallazgo de un nuevo lago subglacial en la Antártica Occidental por sus científicos. Según su propio sitio web, el hallazgo se reporta en un artículo publicado en "Geophysical Research Letters" el 22 de mayo de 2015 con el título: Subglacial Lake CECs: discovery and in situ survey of a privileged research site in West Antarctica", donde se resalta la importancia de ser el primer lago descubierto por investigadores que no provengan de Europa o los Estados Unidos (Centro de Estudios Cientificos , 2015). Hay que mencionar que el CECs es una corporación de derecho privado, sin fines de lucro, dedicada la investigación científica, y nació como una iniciativa privada en 1984. En un comienzo, su financiamiento provino de las Fundación Tinker, y la Fundación Andes, y posteriormente, recibió el apoyo de la Iniciativa Científica Milenio, una unidad del naciente Ministerio de Planificación de aquel entonces (1990-2005). En la actualidad, el principal financiamiento del CECs, proviene del Programa de Financiamiento Basal de CONICYT, una corporación autónoma dependiente del Ministerio de Educación del Estado de Chile.

Por consiguiente, el descubrimiento del CECs, como resultado de una interacción pública-privada, denota el valor que tiene la ciencia para la diplomacia y la imagen país que construye el Estado dentro del STA. Por lo mismo, la actividad científica no provendrá necesariamente de la iniciativa estatal, ya que una parte importante y significativa se genera desde los ANE, los cuales sintonizan sus intereses con los del Estado, en una relación de beneficio mutuo para, en definitiva, generar actividad científica en el subsistema antártico.

Por último, a modo de proyección para el 2022, cabe mencionar la iniciativa estatal chilena denominada Centro Internacional Antártico, impulsado por el Instituto Antártico Chileno (INACH) y la Universidad de Magallanes, la cual pretende congregar a los científicos del mundo (se calculan alrededor de 500 científicos provenientes de 32 países), con lo objetivos de difundir los temas antárticos y aportar a la industria turística (Emol, 2017). Sin duda, este proyecto dará que hablar debido al impacto económico que va a generar en la región y donde convergerán actores desde distintos sectores públicos y privados de Chile y el extranjero debido a la condición geoestratégica del país hacia la antártica. Sin embargo, es incierto el grado competitividad que tendrá el país, desde sus distintos actores, en las actividades que emerjan de este futuro centro.

\section{Libertad de investigación y bioprospección}

Un caso más sustancial de estas relaciones público-privada en la Antártica, y que ha generado una gran discusión político-jurídica, es sin duda, la bioprospección en el continente, es decir, el conjunto de estudios y búsquedas de organismos y sustancias con potenciales 
usos y beneficios para el ser humano y, sobre todo, con un importante valor comercial para la industria alimentaria, cosmética, farmacéutica y médica. Entonces, valdría preguntarse, ¿Quiénes desarrollan esta actividad científica en el continente blanco? ¿Bajo qué normativa jurídica se regula? ¿A quién pertenecen los beneficios de esta actividad?

Por un lado, son los Estados desarrollados quienes disponen de los recursos financieros, tecnológicos y logísticos para el ejercicio de las actividades de bioprospección. A la vez, asumen por su parte ser los propietarios de las muestras obtenidas en un espacio que jurisdiccionalmente no posee ni reconoce la soberanía política de un Estado u otro. Por otro lado, los Estados en vías de desarrollo, evidentemente, se oponen a esta lógica, ya que los beneficios que se puedan obtener de esta actividad son patrimonio de la humanidad (Villamizar-Lamus, p. 126).

Se puede identificar una lógica pararrealista en los actores que actualmente están conduciendo los proyectos de bioprospección en la Antártica, compuestos por Estados, institutos privados, centro de investigación, universidades y laboratorios de distinto origen y naturaleza, que han puesto su mirada en este continente por los diminutos microorganismos extremófilos.

Estos microorganismos están siendo explotados para investigar nuevos anticongelantes, productos de cirugía y hasta cremas para tratar la piel y las uñas (La Prensa Austral, 2012). El ejemplo más pragmático se materializa en el proyecto Biodiversity of microbial Mats in Antarctica, conocido como Micromats, financiado por la Unión Europea, se compone de distintas entidades a nivel académico, industrial, de investigación y de apoyo logístico, quienes trabajan en conjunto por un interés común que sería, en efecto, la apropiación de derechos de los descubrimientos de prospección biológica. En el caso del proyecto Micromat, el conjunto de actores involucrados se puede desglosar de la siguiente manera:

Del lado de Chile, la directora científica de la Fundación Biociencia, (una organización científica sin fines de lucro), Jenny Blamey, es una de los quince investigadores que componían la expedición científica chilena que en el año 2015 se desplazó a la Estación Polar Científica del continente blanco operada conjuntamente por el Instituto Nacional Antártico de Chile (INACH) y las Fuerzas Armadas, situado a 79 grados de latitud sur (El Siglo de Torreón, 2015). Al igual que la relación entre el CECs y el Estado de Chile, la Fundación Biociencia está produciendo, aunque de forma más reciente y en menor escala, un aporte importante en materia de bioprospección.

Con todo, cabe hacerse el cuestionamiento sobre la existencia de un marco jurídico regulatorio de estas actividades y sobre el derecho de propiedad de los beneficios obtenidos. De acuerdo al principio de libertad de investigación sustentado en el artículo 2 del Tratado Antártico, se ha dado cuenta de un creciente y declarado interés por parte de Estados desarrollados para invertir en estas actividades, levantándose una carrera por la obtención de los títulos de propiedad intelectual de estos descubrimientos y las significativas ganancias que se pueden obtener en la industria. No obstante, el artículo 3, 
Tabla 1. Estamentos vinculados a proyecto "Micromat".

\begin{tabular}{|l|l|}
\hline Estamento & Actor Involucrado \\
\hline Académico & $\begin{array}{l}\text { Universidad de Liège (Bélgica) } \\
\text { Universidad de Gent (Bélgica) } \\
\text { Universidad de Bordeaux (Francia) } \\
\text { Universidad de Nottingham (Reino Unido). }\end{array}$ \\
\hline Industrial & $\begin{array}{l}\text { Merck Sharp \& Dohme (España), } \\
\text { Genencor International y Biosearch —ahora Vicuron- (Italia) }\end{array}$ \\
\hline Investigación & $\begin{array}{l}\text { Deutsche Sammlung von Mikroorganismen und Zellkulturen GmbH (DSMZ) y } \\
\text { Rheinisch-Westfälisches Institut für Wasserforschung Gemeinnützige de Alemania } \\
\text { (IWW); } \\
\text { NERC British Antarctic Survey (BAS) (Reino Unido); } \\
\text { Institute of Royal Netherlands Academy of Arts and Science (Holanda). }\end{array}$ \\
\hline Apoyo Logístico & \begin{tabular}{l} 
Estados de Australia y EE.UU.. \\
\hline
\end{tabular}
\end{tabular}

Fuente: https://cordis.europa.eu/project/rcn/45596_fr.html

establece que "en la medida más amplia posible", las partes contratantes acuerdan proceder al intercambio de información, observaciones y resultados de la investigación científica, los cuales estarán disponibles libremente (Tratado Antartico, 1959) pero ¿hasta qué punto los resultados de estas investigaciones están realmente disponibles para la comunidad científica internacional, si el acto de registro de patentes, significa en sí mismo la protección de una idea o descubrimiento?

Según Villamizar, un estudio de la Universidad de las Naciones Unidas, entre 1988 y 1989 había una sola patente de invención procedente de la bioprospección antártica, mientras que para octubre de 2013 la página web www.bioprospector.org registra 185 patentes, y el Information Paper de la XXXVI RCTA (Bruselas, 2013) da cuenta de al menos 439 especies antárticas en patentes (Villamizar-Lamus, 2015, p. 316). Sin duda alguna, estas actividades generan beneficios exclusivos de acuerdo a la obtención de los derechos de propiedad, de ahí que nace la discusión sobre el alcance jurídico del STA, y, aunque se asume que las actividades de bioprospección pueden generar aportes a la humanidad, la apropiación de sus resultados en un espacio de gobernanza internacional sustentado en un tratado que "garantiza" el libre acceso a dicha información, lo hace una actividad contraproducente a la naturaleza del mismo.

\section{Medioambiente, Estados y Actores no estatales en la Antártica}

En relación a los ANE vinculados a la protección y conservación del medio ambiente antártico, estos se constituyen principalmente por Organizaciones No Gubernamentales (ONG) de pequeño, mediano y alto alcance, dentro de las cuales se destacará el caso de la 
Antarctic Southern Ocean Coalition ${ }^{3}$, que cuenta desde 1991 con la condición de miembro observador permanente en las RCTA, informando, recomendando y evaluando las distintas actividades que se realizan en el continente.

Esta organización, tiene su origen en los años 70 y 80 cuando la inestabilidad de las actividades petroleras del mundo amenazaban el ecosistema ${ }^{4}$ de la Antártica, y las partes consultivas del Tratado Antártico, querían dar luz verde a la posibilidad de explotar los recursos minerales del continente bajo la normativa que se denominó Convención para la Reglamentación de las Actividades sobre Recursos Minerales Antárticos (CRAMRA), coyuntura que condujo a la movilización de organizaciones civiles y audaces líderes de la ciencia y la política, fraguándose un proceso de concientización sobre los impactos medioambientales que podría generar tal tentativa.

Una de las primeras reacciones fue el posicionamiento en el continente de la ONG Greenpeace, transformándose en la única organización no estatal en tener una base de estudios científicos entre 1987 y 1991 (Greenpeace, 2015) llamada World Park Base, desde donde realizaron inspecciones a otras estaciones en las regiones del Mar de Ross y la Península Antártica. Del mismo modo, protestaron contra el Estado francés que pretendía la construcción de una pista de aterrizaje en Dumont d’Urville y, así también, contra la caza japonesa de ballenas. No obstante, fuera del continente, estaba ASOC, ONG fundada por James Barnes en 1978, quien se dio cuenta que las Partes consultivas del Tratado Antártico estaban negociando "en secreto" " un marco para la prospección de minerales y gas en la Antártida, e instó a este abogado a la conformación de esta organización con 3 propósitos claros:

3 Contribuyó en tres ámbitos claros: dar marcha atrás a la Convención de Wellington del 2 de julio de 1988. instar en la implementación del Protocolo al Tratado Antártico sobre Protección del Medio Ambiente de 1991. validar la voz de los ambientalistas en las RCTA como observadores.

4 Primero en 1973 la organización de países árabes exportadores de petróleo, junto a los miembros árabes de la OPEP decidieron suspender las exportaciones de petróleo a los países que apoyaron a Israel en la guerra de Yom Kipur. Después en 1979 tras los efectos de la revolución iraní y la guerra Irak-Irán los precios se dispararon nuevamente, y la posterior caída de los precios del petróleo crudo en los años 80, conduciría a los miembros, sobre todo a occidente, a voltear su mirada a la región antártica.

5 De este modo nació la idea de conformar una campaña para convertir a la Antártica en un World Park, idea que surgió en el año 1979 por la ONG Greenpeace de cara a la amenaza de la explotación de los recursos minerales, sin embargo, las reglas de tratado antártico establecen que para poseer derecho a voz dentro del STA era necesario poseer una base en el continente. Esta base constituiría un acto de presencia tacita en el territorio lo que otorgaría la autoridad para que la ONG pudiera tener derecho a voz y permitiría cuestionar los reclamos territoriales nacionales con el argumento de que la Antártida debería conservarse como un bien común global, no perteneciente a nadie. Ninguna organización no gubernamental había establecido una base en la Antártida; hubo muchos obstáculos, tanto políticos como prácticos. En 1987, el MV Greenpeace atracó en la Antártida (después de que el tiempo había detenido su primer intento) y unas semanas después, la Base del Parque Mundial estaba en funcionamiento. Los intrépidos pioneros de Greenpeace se quedaron desde 1987 hasta 1991.

$6 \quad$ ASOC logró sacudir este sistema al obtener una copia secreta de uno de los primeros borradores, que distribuyeron públicamente. Al informar a países en desarrollo como Malasia, India y Brasil sobre las negociaciones secretas, las organizaciones miembros de ASOC pudieron iniciar debates en las Naciones Unidas. Los activistas también llamaron la atención sobre las prácticas descuidadas de eliminación de desechos en las estaciones de investigación científica, y los planes franceses de dinamitar varias islas antárticas y desplazar a muchas colonias de pingüinos para construir una pista de aterrizaje. 
1. Convencer a los gobiernos para la negociación de un tratado de pesca mundial "ecosistémico". (antesala de la CCAMLR)

2. Prevenir la explotación de minerales en la Antártida al bloquear el Convenio de Minerales

3. Abrir el Sistema del Tratado Antártico para las ONG y organismos internacionales especializados.

De este modo, ASOC se propuso romper con el realismo imperante del STA para cooptar a los Estados en la adopción de medidas para la protección y preservación de los ecosistemas antárticos. Para ASOC, fue un triunfo la ratificación de la CCAMLR en 1981 y a pesar que las negociaciones del CRAMRA seguían adelante, en 1989, los Estados de Francia ${ }^{7}$ y Australia ${ }^{8}$ no ratificaron la convención, debido principalmente a la presión que generó la opinión pública internacional, y la movilización de otros ANE como Jacques Cousteau, el príncipe Sadruddin Aga Khan y Ted Turner (Greenpeace, 2015). Estos países declararon la explotación minera incompatible con la protección del medioambiente del continente y el proceso fue bloqueado (Velcof, 2009, p 73). De este modo, en 1991 los miembros del Tratado Antártico adoptaron un Protocolo de protección al medioambiente, con una prohibición de 50 años para toda explotación minera. Pero no menos significativo, es que el mismo año, ASOC obtuvo la condición de miembro observador en el STA, como la voz de las ONG, ampliando su área de acción hacia la ciencia, el turismo, el transporte marítimo y el cambio climático, presentando informes en nombre de las 17 organizaciones miembros que trabajan con el personal de la Secretaría y el equipo de campaña, para desarrollar y promover los objetivos de conservación antárticos (ASOC, 2018).

En entrevista directa con Claire Christian9, directora de ASOC, ella considera que muchos países están orgullosos de haber sido capaces de dar un paso visionario en nombre del mundo. Afirma que existe un gran sentido de orgullo y de un liderazgo nacional; que se tomó una decisión que muchos consideraban no ser práctica en el momento, pero ahora muchas personas consideran que es un gran logro ambiental y existe un deseo de no borrar ese logro. (C. Christian, comunicación personal, 13 de febrero 2018).

7 El primer ministro francés de la época, Michel Rocard, dotado él mismo de una cultura y de una sensibilidad medioambientalista, fue receptivo a los argumentos de los ANE defensores de la Antártica, y fue el primero en Francia en declarar que "la Francia no firmará la convención de Wellington en las condiciones presentes."

8 En Australia, las elecciones de 1988 habían puesto en evidencia la subida de los Verdes (The Greens) australianos, donde habían obtenido el $20 \%$ de los sufragios. El primer ministro australiano, Bob Hawke, estimó que la prohibición de la explotación minera en la Antártica era un medio para recuperar votos de cara a las elecciones de 1990 (Velcof, 2009).

9 Claire Christian ha trabajado para ASOC desde 2009 y ha desarrollado experiencia en toda la gama de cuestiones relevantes al medioambiente antártico. En su trabajo con ASOC, desarrolla políticas y estrategias relacionadas con muchos de los problemas que afectan el medio ambiente antártico, incluido el turismo, la pesca y el cambio climático. Se relaciona con una gran variedad de partes interesadas de la Antártida, desde funcionarios gubernamentales hasta científicos investigadores. Claire recibió un M.A. en Asuntos Internacionales en la American University, School of International Service en mayo de 2008. Anteriormente trabajó como Asistente de Programa en el Consejo Nacional de Ciencia y Medio Ambiente en Washington, DC. 
Además, en relación al rol que asumen los ANE en la política internacional, Christian afirma que los gobiernos no tienen como prioridad la Antártida, y estos pueden ser muy fuertes en asuntos ambientales, por ejemplo, en la protección de los bosques, es decir, en un área determinada. Sin embargo, no existe un mandato desde las autoridades para trabajar en la protección de la Antártida. De este modo, según Christian, la ventaja que poseen los actores de la sociedad civil y los Actores No Estatales, es que están enfocados en sus áreas de experticia, y no están limitados por otras prioridades, por lo tanto, estos son capaces de ayudar a mantener el sistema centrado atendiendo cuestiones específicas con políticas específicas. (C. Christian, comunicación personal, 13 de febrero 2018).

A modo de ejemplo, en el año 2013, en la XXXVI RCTA en Bruselas, la ASOC presentó el IP (documento de información) 64 Biological prospecting and the Antarctic environment, que examina la prospección biológica desde la perspectiva de su impacto medioambiental. La ASOC se mostró preocupada por el hecho de que la bioprospección estaba actualmente sin regular y recomendó que las Partes declarasen cualquier intención de realizar actividades de prospección biológica al presentar información mediante el Sistema Electrónico de Intercambio de Información (SEII), identificasen los posibles impactos medioambientales en evaluaciones de impacto ambiental (EIAs) y que vigilasen los efectos de tales actividades en el medioambiente. Además, la ASOC recomendó que se establezca un mecanismo adecuado para identificar la captura de recursos marinos vivos en el Océano Austral, relativas a actividades de prospección biológica (Secretaría del Tratado, 2013, p. 90).

Actualmente, la sociedad civil sigue movilizando recursos y ejerciendo influencia dentro del STA. En efecto, el actor de cine Leonardo DiCaprio solicitó en octubre del año 2012 en una conferencia en Australia, la creación de un santuario marino en el Antártico para proteger a las especies marinas por medio de un mensaje por correo electrónico al grupo de defensa de la naturaleza Avaaz ${ }^{11}$ durante una reunión de la CCAMLR, el actor pidió "un impulso de la gente" para crear este santuario (La Nacion, 2012). El grupo de campaña Avaaz.org recibió más de 2 millones de firmas y su director de campaña, Luis Morago, afirmo que hay un impulso masivo en el mundo para proteger nuestros océanos. De este modo, tras complejas negociaciones, la CCAMLR anunció en octubre del 2016 el establecimiento de dicha área que gestiona la pesca en el océano Austral como parte del sistema del Tratado Antártico. El periódico The Guardian publicó: "Los gobiernos acaban de establecer el objetivo histórico de proteger el 30\% de nuestros océanos, y millones de personas en todo el mundo están presionando para obtener más áreas protegidas para lograr ese objetivo. El Mar de Ross es solo el comienzo" (Slezak, 2016).

Pareciera, que los llamados que hizo Kofi Annan durante su mandato de la ONU (19972006) tuvieron un efecto cuando invitó a la sociedad civil (a los pueblos como él decía) a participar en la vida internacional en un sentido político y ambiental. Así, se entabla una

11 Es una organización civil a escala global con sede en Estados Unidos. Fue fundada en el año 2007 y promueve el activismo ciudadano a través de internet. Su principal fuente de financiamiento proviene de las colaboraciones de sus miembros. 
red para las comunicaciones entre los Estados y la sociedad civil, donde se articulan puntos de vista para influir en las instituciones políticas, adquiriendo considerable popularidad y legitimidad, asumiendo frente a los organismos internacionales un rol de representación de intereses con efectos ya tangibles en la práctica (Allard, 2008, p. 29).

\section{Turismo y geopolítica en la Antártica. El rol de los actores no estatales}

El turismo en la Antártica es una de las actividades que ha tenido más revuelo en los últimos años, y gran parte de los expertos consultados para esta investigación se refieren a la gran incertidumbre que genera esta actividad debido a los intereses geopolíticos y pararrealistas que implican la creciente visita de turistas. Como bien se sabe y también se puede percibir, los grandes avances en tecnología, transporte y telecomunicaciones ha permitido que esta actividad haya aumentado exponencialmente, con importantes ganancias para las empresas transnacionales que la promueven.

Por otro lado, la creciente visita de turistas asume ciertos riesgos en el medioambiente y los espacios preservados del continente. Además, el turismo en el Antártica, es en sí una expresión de intereses privados, la cual podría transformarse, según Strobel y Frank (Strobel \& Frank, 2007, p. 167), en una contienda geopolítica o, al menos, en un factor geopolítico y como fuente de rivalidades, particularmente en la península antártica que es la región más accesible del continente donde Chile y Argentina tienen una ventaja geoestratégica.

En el mismo sentido, la International Association of Antarctica Tour Operators (IAATO), organización creada en 1991 que promueve los intereses de los turoperadores en la Antártica, está compuesta por más de 100 empresas privadas de Argentina, Australia, Bélgica, Canadá, Chile, Francia, Alemania, Italia, Japón, Holanda, Nueva Zelanda, Noruega, República Popular de China, Rusia, Sudáfrica, Suecia, Suiza, Reino Unido y Estados Unidos (IAATO, 2018). Esta organización que se encuentra presente en la RCTA como observadora, y además ejerce presión como fuerza transnacional, para promover o evitar la adopción de medidas que puedan beneficiar o perjudicar los intereses de la actividad turística. Según Strobel y Frank, la IAATO se apoya en los Estados anglosajones, generalmente más favorables que los otros al turismo en la región Antártica (Strobel \& Frank, 2007, p. 175), lo cual sea muy probable debido que gran parte de las empresas dedicadas a esta actividad tienen su casa matriz en estos países (EE.UU., Canadá, Reino Unido, Australia)

En efecto, en la temporada 1991-1992, cuando se fundó la IAATO, aproximadamente 6.400 turistas visitaron la Antártida, viajando a bordo de diez barcos de expedición manejados por seis operadores más las actividades terrestres del único operador de tierra. Los nuevos miembros continuaron uniéndose a un ritmo constante (IAATO, 2018). Según la IAATO, el principal impulso detrás de la formación de una asociación industrial global sin "fines de lucro" fue doble. En primer lugar, la firma del Protocolo Ambiental Antártico a principios de 1991 por las naciones del Tratado Antártico estableció estándares rigurosos 
más allá de los ya delineados en el Tratado Antártico de 1959, que designaba a la Antártida como "reserva natural dedicada a la paz y la ciencia" y el Protocolo proporcionó el marco para la protección continua del medioambiente antártico (IAATO, 2018). En segundo lugar, al unirse, las empresas pudieron aunar recursos y presionar a sus gobiernos nacionales para desarrollar regulaciones y pautas sustantivas compatibles con las mejores prácticas que ya se estaban siguiendo en el campo (IAATO, 2018).

Sin embargo, el turismo en Antártica no es una práctica abierta y en disposición de toda la población mundial, ni siquiera los países más cercanos como Chile y Argentina aparecen en las últimas estadísticas de visitantes, en contraste a estadounidenses, chinos, australianos y europeos, que, según los informes de la IAATO, son significativamente más numerosos. A lo anterior, hay que considerar que existe una relación directa entre el país al cual pertenece la empresa turística y la nacionalidad misma de los visitantes que consumen los traslados y servicios de cada operador y, por otro lado, los millonarios costos por los servicios de esta industria, marginan a los pequeños y medianos países del sistema, que sin tener una oferta local adaptada a su realidad nacional, prácticamente quedan fuera de la actividad, sin importar si están geográficamente más cerca del continente.

A modo de ejemplo, el registro estadístico que dispone la IAATO de la temporada 1994-1995, que con un total de 8.210 visitantes 36\% eran estadounidenses, 18\% alemanes, 7.3\% argentinos, 5.3\% ingleses, 4\% brasileños, 4\% australianos y 3\% japoneses. Trece años después, durante la temporada 2007-2008, con un total de 46.069 visitantes, 35\% eran estadounidenses, 16\% ingleses, 11\% alemanes, 7\% australianos y 6\% canadienses. En la última temporada 2017-2018, con un total de 51.131 visitantes, el 33\% son estadounidenses, el 14\% chinos, 11\%australianos, 9\% ingleses $8 \%$ alemanes $5 \%$ canadienses, y $4 \%$ franceses, que viajan por medio de empresas del mismo país (IAATO, 2018).

Para el caso de los emergentes y numerosos visitantes chinos, el potente crecimiento de su economía de los últimos años, ha permitido a sus nacionales acceder a las ofertas turísticas de otros países. No obstante, una noticia del año 2017 recorrió el mundo afirmando que China está afianzando su presencia en la Antártida con su primer vuelo comercial (El Mundo, 2017), permitiendo a sus pasajeros llegar hasta el polo sur, el hecho fue calificado como un "hito", ya que libera a los chinos de que recurrir a los servicios extranjeros para poder llegar a este destino, siendo la única posibilidad con la que contaban hasta la fecha (El Mundo, 2017). A modo de proyección, esta dinámica se mantendrá en los años próximos años, junto a la emergencia de empresas turísticas chinas en la Antártica, propiciado esencialmente por la política polar que ha implementado este país para las nuevas rutas comerciales de los mares descongelados del norte, y el mantenido levantamiento de bases en la Antártica. En efecto, desde el 2013, China ha identificado las regiones polares como una de las nuevas fronteras estratégicas del país, lo que se traduce en una fuerte voluntad política en formar parte del cómo se gobernarán estos territorios en el futuro. Para ello, el reciente Congreso del Partido Comunista estableció en su nuevo plan quinquenal que el Gobierno invertirá grandes 
cantidades de recursos en proyectos de exploración de los dos polos. "Con el tiempo, las ambiciones chinas contemplan poder presentar su propia propuesta para influir en cómo se gobernarán las dos regiones polares" (El Mundo, 2017).

Por otro lado, desde Latinoamérica como actor, se puede considerar la iniciativa del gobierno argentino de Mauricio Macri para iniciar vuelos comerciales a la Antártica desde el 2018 (El País, 2016), que seguramente será un trabajo en conjunto entre públicos y privados, del mismo modo, como sucedió con el cuestionado -principalmente por Greenpeace- evento musical, de la banda estadounidense Metallica, que realizó un concierto en la base argentina Marambio en el año 2013, en un proyecto colaborativo entre el Estado argentino, la productora Plataforma TV y la multinacional Coca-Cola. El proyecto titulado Música Zero, se atribuyó a sí misma la clasificación de "actividad de turismo no gubernamental" según recomendación 1 de la XVIII RCTA del año 1994, y siguiendo los Principios Generales del Turismo Antártico de resolución 7, de la XXXII RCTA del año 2009 (eneAmbiental, 2013).

Hay que considerar que el turismo posee un marco regulatorio de acuerdo al protocolo del medioambiente, por lo mismo existe una latente preocupación por los efectos que podría contraer la actividad turística en la Antártica. Por ejemplo, en agosto del año 2011, se prohibió el uso y transporte de fueloil pesado, lo que tuvo un impacto significativo en los buques de pasajeros que realizan viajes de tipo "cruceros". Además, según Osvaldo Urrutia ${ }^{12}$, existe una regulación que se traslapa con diversos regímenes jurídicos, un buque de turismo está sujeto a las reglas de la Organización Marítima Internacional (OMI), más las reglas que impone el mismo tratado antártico (O. Urrutia, comunicación personal, 18 de enero de 2018) -como el conocido Código Polar que estableció la OMI a principios del 2017- para regular las actividades de las embarcaciones en aguas polares. Aun así, la mayoría de los entrevistados para este trabajo, concuerdan en que no se sabe en qué medida la regulación de esta actividad es efectiva en la práctica.

Por otro lado, en cuanto a los lugares más visitados del continente, el caso del Glaciar Unión es un buen ejemplo de colaboración entre las instituciones de un Estado y las empresas transnacionales de turismo. Este lugar fue, según la IAATO, durante la temporada 2016-2017, el sitio más popular para los visitantes en el espacio continental. Lo curioso, es que en este lugar se encuentra en una zona que solo está reclamada por Chile y en noviembre del 2014, el gobierno de Sebastián Piñera, inauguró la Estación Polar Científica Conjunta Glaciar Unión, mantenida por el Instituto Antártico Chileno (INACH), la Fuerza Aérea, el Ejército y la Armada de Chile (INACH, 2014). Sin embargo, ya desde noviembre del año 2010 la empresa estadounidense Antarctic Logistics \& Expeditions (ALE) LLC realizaba actividades turísticas en la zona, y desde aquí, inician expediciones privadas hacia el Polo Sur. Tanto el gobierno de Chile como la empresa ALE LLC se trasladaron al lugar después de operar en primera instancia en el Campamento Base Patriot Hills, unos $70 \mathrm{~km}$ al sudeste, donde la compañía privada, antes llamada Adventure Network

12 Profesor de Derecho Internacional, Departamento de Derecho público. Abogado, Pontificia Universidad Católica de Valparaíso, Chile. En el año 2012 fue elegido presidente del Comité Permanente de Ejecución y Cumplimiento de la CCAMLR. 
International, realizaba operaciones desde el año 1987, mientras que la Fuerza Aérea de Chile (Base Teniente Arturo Parodi Alister) y el INACH (sección denominada Base Antonio Huneeus) mantenían cada uno una base de verano desde 1999. Una prueba del trabajo en conjunto entre el Estado de Chile y ALE LLC, se puede ver en sus informativos turísticos que denotan la disposición de personal local para el ofrecimiento de servicios:

[....] le damos la bienvenida a Chile, su punto de partida hacia la Antártida. Nuestro personal experto habla inglés y español (y algunos otros idiomas también) y está aquí para ayudarlo a prepararse para su próximo viaje. Con más de 20 años de experiencia trabajando y viviendo en Punta Arenas, puedes obtener consejos directamente de los 'locales': algunos miembros de nuestro equipo nacieron y se criaron aquí. Te ayudaremos a comprar esos artículos de último minuto, rastrear el equipaje perdido y podemos decirte quién sirve los mejores piscos sours de la ciudad. Nuestro objetivo es hacer que su tiempo en Punta Arenas sea lo más sencillo posible. (ALE, 2018).

Así y todo, el trabajo en conjunto no ha generado un aumento en las visitas de turistas chilenos, se cree, principalmente a la inexistencia de una oferta turística nacional más adecuada a la realidad de sus nacionales. Además, aún quedan vacíos en el desarrollo de una conciencia Antártica en los planes educativos del país, y gran parte de los asuntos antárticos están bajo la dirección de las fuerzas armadas, y se proyecta poco hacia y desde la sociedad civil.

Resulta muy interesante la visión del Dr. Luis Valentín Ferrada ${ }^{13}$, en entrevista directa con él, concibe que el turismo es algo que hay que repensar y considera que esta actividad funciona con muy poca regulación internacional, y con débil capacidad para sancionar efectivamente si alguien infringiera las normas del sistema (L. Ferrada, comunicación personal, 05 de enero 2018). Efectivamente, el turismo es una actividad que podría considerarse como contra naturam, es decir, que difícilmente se puede asociar la creciente y lucrativa actividad turística con la protección y preservación del medio ambiente antártico. En el mismo sentido, Ferrada expresa su parecer sobre esta controversial relación:

[....] el turismo funciona básicamente bajo la regla de la autorregulación que podría ejercer la IATTO, la cual vende aventuras antárticas en un medio ambiente prístino, siendo ellos los principales partidarios que ese ambiente prístino perdure en el tiempo. Esto no es convincente en lo más mínimo, ya que las empresas privadas tienen como regla la maximización de las utilidades, y está bien, ese es el mundo de la empresa privada, ganar cada vez más que el año anterior, el punto, es que esa lógica no parece que sea muy compatible con una preocupación efectiva del medio ambiente en la Antártica. (L. Ferrada, comunicación personal, 05 de enero 2018).

13 Profesor de Derecho Internacional en la Facultad de Derecho de la Universidad de Chile. Fue encargado de temas de Derecho Internacional Público y materias antárticas en la Subsecretaría de Defensa, Ministerio de Defensa Nacional y asesor en materias de Derecho Antártico en el Ministerio de Relaciones Exteriores. 
En definitiva, el turismo antártico se presenta como uno de las actividades más cuestionadas debido al impacto ambiental que podría generar en el continente. Si bien, hoy por hoy, hay más de 50.000 personas visitan la antártica con fines turísticos, se espera que está cifra siga creciendo ¿Cuáles son los limites? Esta es una pregunta que es difícil responder si se considera que las empresas turísticas tienen como objetivo el incremento permanente de sus ganancias temporada tras temporada, por lo tanto, existe una contradicción por parte de los turoperadores en su intento de conciliar el apogeo del turismo con la preservación del carácter natural y salvaje de la Antártica. (Strobel \& Frank, 2007).

\section{CONCLUSIONES}

Se ha visto, que los ANE en el STA tienen una significancia de poder en lo políticoterritorial, lo ideológico, lo social-global y en lo económico, la que se materializa en actividades científicas, de turismo y medioambientales (sin considerar, en el presente artículo, el área de la pesca, los transportes y las telecomunicaciones). Estas organizaciones independientes de los Estados, pero a la vez muy ligadas a ellos, pueden poseer o no fines de lucro, pero lo más relevante es que los ANE, son actores con una capacidad más receptiva y con cualidades que los Estados no manejan, lo que se traduce en una mayor sensibilidad a los cambios y la tecnología, de este modo, se configuran relaciones complementarias ante objetivos políticos, territoriales, ideológicos o económicos comunes.

En plena concordancia con la historiadora antártica Consuelo León ${ }^{14}$ (C. León, comunicación personal, 22 de enero de 2018), los ANE trabajan en sintonía con las normas del Tratado Antártico, el cual permite el desarrollo de actividades pacificas en el continente, quienes, a pesar de no ser sujetos de derecho internacional, ya que son solo los Estados y las organizaciones intergubernamentales, funcionen como actores del sistema, con una progresiva y creciente importancia en el continente.

Efectivamente, tal como se ha expuesto en el presente trabajo, los ANE poseen un grado de autonomía que depende del tamaño de estas organizaciones, las mas pequeñas deben someterse al control de algún Estado miembro, sin embargo, las grandes poseen mayores recursos técnicos, humanos y financieros, para el desplazamiento y desarrollo de sus actividades. Se puede ver que las grandes empresas, principalmente ligadas al turismo y la ciencia, tienen una capacidad de acción que otros Estados medianos no, representando un inminente peligro, ya que difícilmente deben someterse a los mecanismos de control del sistema antártico.

Se ha visto que la arista de la ciencia, como principal actividad del continente, ha devenido, en un proceso histórico, un instrumento de interacción diplomática entre los distintos agentes del sistema. Permite que se generen relaciones entre el sector público y privado, invirtiendo recursos en la producción de información científica, desde publicaciones

14 Historiadora e investigadora FONDECYT, especialista en relaciones internacionales e historia antártica y hemisférica. Presidenta de la Fundación Valle Hermoso y directora de la Revista Estudios Hemisféricos y Polares. Viña del Mar. Chile. 
de working papers, hasta la prospección, exploración, extracción y selección de organismos vivos para la industria biotecnológica. Lo anterior, representa intereses complementarios para los Estados y los privados, para los primeros, la obtención de un liderazgo y protagonismo en las relaciones interestatales, y para los segundos, la obtención de utilidades.

Por otro lado, los ANE medioambientales han desarrollado un importante liderazgo y capacidad de acción para establecer lineamientos de protección y preservación del frágil ecosistema antártico. Se muestran como la parte contrapuesta de las actividades antárticas, observando, evaluando y denunciando sus impactos ambientales. Se dejó en evidencia que el giro en $180^{\circ}$ que tuvieron los Estados para prohibir, o por lo menos detener, la CRAMRA, fue una victoria principalmente para estas organizaciones. En efecto, la capacidad de acción que tuvo Greenpeace junto al liderazgo de ASOC dentro de las RCTA, sumado a la fuerza movilizadora de figuras públicas, refuerza la idea que la sociedad civil global ha tenido, y tiene, la capacidad de cargar la balanza en las decisiones del sistema antártico.

Por su parte el Turismo, tienen una clara connotación geopolítica-económica, puesto que las cifras no favorecen a los países que están geográficamente más cerca del continente, y si a aquellos Estados que han desarrollado, por parte de sus privados nacionales, una industria turística más potente, y deja ver el influjo de las empresas a través de la IAATO en las RCTA. En entrevista con Anne Choquet,, ${ }^{15}$ (A. Choquet, comunicación personal, 07 de marzo 2018), experta en derecho polar, se concuerda que el posicionamiento en la Antártica de los ANE es real. Ellos tienen la posibilidad de presentar información participando en las negociaciones, donde incluso no se ve una diferencia entre el compartimento de un Estado no consultivo y una ONG, por lo tanto, son mas que meros observadores. Según Choquet, la riqueza de las discusiones en las RCTA tiene su núcleo en la diversidad de actores presentes, por lo tanto, la capacidad de influencia de los ANE puede igualmente verse en la presencia de personas que están a veces en una delegación de Estado u ONG como la IAATO. No es muy visible, pero su presencia puede constatarse.

Con todo, se considera que los ANE, en todas las dimensiones expuestas, son actores de injerencia, por lo tanto, el enfoque pararrealista propuesto, se muestra más adaptado a la hora de entender las dinámicas de poder en interacción en el continente. Efectivamente, el conjunto de áreas y actividades en las cuales influyen los ANE, les permite cooptar, aunque sea de forma relativa, a los distintos actores del STA. Por consiguiente, hoy, en el siglo XXI, se hace necesario repensar la forma en que entendemos la relaciones inter y transnacionales en la Antártica, y no resulta asertivo entenderlas por separado, sino que existen intersecciones donde convergen intereses comunes y contrapuestos. Las relaciones entre Estados, principalmente los desarrollados, y ANE se intensifican, conformando asociaciones, movilizando recursos en favor de intereses geopolíticos a través de la presencia y protagonismo de agentes nacionales ligados a la protección y preservación de los ecosistemas y la promoción de actividades

15 De nacionalidad francesa, Anne Choquet es académica e investigadora en derecho en Brest Business School. Posee un doctorado sobre la protección del medioambiente antártico. Desarrolla trabajos sobre el derecho de las regiones polares, tanto en materia de protección del medioambiente como en gestión de actividades humanas. 
científicas, turísticas y otras materias como la pesca, los transportes y las telecomunicaciones. Por lo tanto, existe una complementariedad que es tacita, es decir, que probablemente nunca será oficial, sino que se supone y se sobreentiende. A modo de proyección, los Estados seguirán jugando un rol formal bajo las RCTA, pero se presume que las relaciones interestatales en el STA estarán cada vez más permeadas por la influencia de los ANE como resultado de los procesos de globalización, sin duda, esta realidad genera incertidumbre por el avenir del continente y la prevalencia de los propósitos y principios del Tratado Antártico.

\section{REFERENCIAS}

ALE. Antarctic Logistic Expeditions. (2018). Our Team. Disponible en: https://antarcticlogistics.com/about-ale/our-team/punta-arenas-team/

Allan, P. (2001). Ontología y explicaciones en la Teoría de las Relaciones Internacionales. Ciencia Política, 21(1), 77-106.

Allard, R. (2008). La globalización por dentro. Santiago: Catalonia Editores.

Antártico, T. (1959). Tratado Antártico. Washington.

Añaños, M. (2014). La idea de los bienes comunes en el sistema internacional: ¿renacimiento o extinción? UNISCI Discussion Papers, 34, 153-178.

Arana, I. (18 de diciembre de 2017). El Mundo. China afianza su presencia en la Antártida con su primer vuelo comercial. Disponible en: https://www.elmundo.es/ciencia-y-salud/ ciencia/2017/12/18/5a37f45f468aeb42068b469e.html

Barbé, E. (2007). Relaciones Internacionales. Madrid: Tecnos Editores.

Barreiro, R. (26 de julio de 2016). El País. Argentina anuncia vuelos comerciales a la Antártida. Disponible en: https://elpais.com/internacional/2016/07/26/ argentina/1469554639_740377.html

Centro de Estudios Científicos. (22 de mayo de 2015). Científicos del CECs descubren nuevo lago subglacial en la Antártica Occidental. Disponible en: http://www.cecs.cl/ website/index.php/es/menu-noticias/1092-cientificos-del-cecs-descubren-nuevo-lagosubglacial-en-la-antartica-occidental

CIA (2010). The CIA World Factbook. EEUU: Ed. Skyhorse Publishing, Inc.

ASOC (1 de marzo de 2018). Antarctic and Southern Ocean Coalition. Disponible en: https:// www.asoc.org

Duarte Villa, R. (2001). Atores não-estatais e meio ambiente nas relações internacionais: Greenpeace e a Antártica. Desenvolvimento e Meio Ambiente, 4, 45-57.

Dudeney, J., \& Walton, D. (2012). Leadership in politics and science within the Antarctic Treaty. Polar Research, 31(1), 1-9.

El Siglo de Torreón. (26 de diciembre de 2015). Los secretos de los extremófilos en la Antártida. Disponible en: https://www.elsiglodetorreon.com.mx/noticia/1182325.los-secretosde-los-extremofilos-en-la-antartida.html 
Emol (24 de abril de 2017). Chile tendrá en 2022 un Centro Antártico Internacional para 500 investigadores. Disponible en: https://www.emol.com/noticias/ Tecnologia/2017/04/24/855397/Chile-tendra-en-2022-un-Centro-AntarticoInternacional-para-500-investigadores.html

EneAmbiental Consultoría (2013). Informe de evaluación Medioambiental Inicial. Proyecto “Música Zero” Base Carlini. La Plata, Buenos Aires. Disponible en: https://docplayer. es/13274998-Evaluacion-medioambiental-inicial.html

Gilpin, R. (1981). War and change in world politics. Cambridge. Cambridge University Press. Greenpeace (06 de Junio de 2015). Creating the World Park Antarctica. Disponible en: https:// www.greenpeace.org/usa/victories/creating-the-world-park-antarctica/

Hemmings, A. (2014). The Changing Face of Non-State Actors in Antarctica. Antarctic, 32 (2), 30-33.

IAATO. (Octubre de 2018). Who is IAATO? Disponible en: https://iaato.org/who-is-iaato https://iaato.org/history-of-iaato

INACH, M. e. (6 de enero de 2014). Presidente Piñera inaugura Estación Polar Científica Conjunta “Glaciar Unión”. Disponible en: http://www.inach.cl/inach/?p=10106

La Nación, D. (23 de octubre de 2012). Leonardo DiCaprio pide la creación de un santuario marino en el Antártico. La Nación, pág. 1. Disponible en: https://www.nacion.com/ viva/leonardo-dicaprio-pide-la-creacion-de-un-santuario-marino-en-el-antartico/3EC2V 6XHW5CBBPOM5OWCKWLCJ4/story/

La Porte Fernández, T. (2016). Influencia de los Actores Internacionales no-estatales en las Estrategias Diplomáticas: consideraciones desde la comunicación pública. Comillas Journal of International Relations, 6, 28-39.

La Prensa Austral (13 de marzo de 2012). El quien es quién en la Antártica: las naciones que la reclaman y las que están. La Prensa Austral.

Rothwell, D. (2009). The IPY and the Antartctic Treaty System: Reflections 50 Years Later. En Legacies and Change in Polar Sciences. Shadian J., Tennberg, M., England: Ashgate Editores.

Secretaría del Tratado, A. (2013). Informe Final de la Trigésima Sexta Reunión Consultiva del Tratado Antártico. Buenos Aires. Disponible en: https://www.ats.aq/documents/ ATCM36/fr/ATCM36_fr001_s.pdf

Slezak, M. (28 de octubre de 2016). World's largest marine park created in Ross Sea in Antarctica in landmark deal. The Guardian, pág. 1. Disponible en: https://www.theguardian.com/ world/2016/oct/28/worlds-largest-marine-park-created-in-ross-sea-in-antarctica-inlandmark-deal

Strobel, M., \& Frank, T. (2007). Le tourisme en Antarctique : un enjeu géopolitique ? Hérodote (127), 167-177.

Velcof, J. (2009). Le Statut International de l'Antarctique. Séminaire « Droit international public » Sous la direction de M. Moncef Kdhir. Lyon : Institut d’Etudes Politiques de 
Lyon. Université Lyon 2.

Villamizar Lamus, F. (2015). Bioprospección antártica: ¿hacia una institucionalidad jurídica sui géneris? Vniversitas, 309, 309-346.

Villamizar Lamus, F. (2017). Más allá de las fronteras: los horizontes geopolíticos de la Antártida. Bogotá: Fondo Editorial Ediciones Universidad Cooperativa de Colombia, Editores. 
